\title{
Preface to Special Issue on Neutron Scattering for Catalysis
}

\author{
Zili Wu$^{1} \cdot$ A. J. Ramirez-Cuesta ${ }^{2} \cdot$ Katsuyuki Fukutani ${ }^{3}$
}

Accepted: 12 July 2021 / Published online: 21 July 2021

(c) The Author(s), under exclusive licence to Springer Science+Business Media, LLC, part of Springer Nature 2021

In the pursuit of understanding the chemical transformations of catalytic reactions and the structural evolution of catalysts at the molecular level, a suite of advanced experimental methods has been developed in the studies of catalysis science. Among these methods, neutron scattering not only gives catalytic information that is highly complementary to other microscopic scattering techniques such as electrons (microscopy and diffraction) and photons from visible light to synchrotron $\mathrm{X}$-rays, but also frequently provides unique insights into catalysis regarding the structure and chemical nature of light elements such as hydrogen and oxygen that are either not possible or difficult to study with other approaches.

Although the use of neutron techniques to studying catalytic processes is still scarce compared to X-ray, the development and application of neutron scattering techniques in catalysis have achieved noticeable advances, which motivated us to put together a special issue dedicated to "Neutron Scattering for Catalysis". This special issue contains 14 invited papers, including 4 reviews and 10 research articles, covering the most commonly utilized techniques. Inelastic neutron scattering (INS) is powerful in identifying the chemical nature of hydrogen-containing species while their structure and dynamic behavior can be revealed via neutron diffraction, and quasi-elastic neutron scattering (QENS) approaches. In the following, we provide a brief description

\section{Zili Wu}

wuz1@ornl.gov

A. J. Ramirez-Cuesta ramirezcueaj@ornl.gov

Katsuyuki Fukutani

fukutani@iis.u-tokyo.ac.jp

1 Oak Ridge National Laboratory, Chemical Sciences Division and Center for Nanophase Materials Sciences, Oak Ridge, TN, USA

2 Oak Ridge National Laboratory, Neutron Sciences Division, Oak Ridge, TN, USA

3 Institute of Industrial Science, University of Tokyo, Tokyo, Japan of the papers included in this issue as an outline to encourage further reading.

The status of surface species on solid catalysts during heterogeneous catalysis is often mysterious. Investigations of the nature of such species are crucial to deconvolute the reaction network and design more efficient catalysts, where INS can play an important role as a vibrational spectroscopy tool. In the mini-review by Yang et al., the applications of INS in two important fields has been described for (i) the behavior of hydrocarbons on metal-oxide and zeolite catalysts and (ii) the formation of hydrocarbonaceous species on methane reforming and Fischer-Tropsch catalysts. Another review demonstrating the power of INS is the characterization of hydrogenous species on/in unsupported and supported palladium by Albers and Parker. This review focuses on identifying and quantifying various hydrogenous species by INS on and inside fresh, hydrogenated, and dehydrogenated palladium black and commerical supported palladium catalysts of different particle size, type of support, and, morphology. These results aid the understanding of hydrogen/ palladium interactions in hydrogen-involved catalytic processes. Not only important in catalysis, but hydrides are also promising hydrogen storage materials. The work by Sato et al. provides an overview of INS studies of hydrogen vibrations in representative hydrides. In particular, mode assignments focused on hydrogen states, local atomic arrangements around hydrogen atoms, hydrogen release reactions, and hydride formation processes based on observed hydrogen vibrations through INS are reviewed. Neutron scattering is also powerful in revealing the crucial roles of hydrogen species played in the physiological functions of biocatalysts-enzymes. In the paper by Higuchi et al., the recent approach towards the neutron structure analysis of a large protein complex, $[\mathrm{NiFe}]$-hydrogenase, was overviewed. They described the preparation of high-quality large protein crystals in $\mathrm{D}_{2} \mathrm{O}$ and neutron diffraction experiments on the large crystals at cryogenic temperature to trap short-lived intermediates in the catalytic reaction.

In the application of INS for understanding surface chemistry in catalysis, Lennon et al. employed INS to study how 
the sodium and sulfur promoted haematite, representative of a candidate Fischer-Tropsch to olefins (FTO) catalyst, behaved differently regarding the nature of carbonaceous deposits after the FTO reaction in comparison to an unpromoted catalyst. Utilizing INS, Borgschulte et al. studied $\mathrm{CO}_{2}$ hydrogenation over a $\mathrm{Cu}$ zeolite catalyst and delivered quantitative information on the product yields of methanol, dimethyl ether, and water during the initial and steady-state of the reaction, and revealed the role of water on the reaction pathways. Lennon and Parker et al. comparatively studied hydrogen interaction with $\mathrm{Ni}$ and Co catalysts in both supported and unsupported forms using INS. They showed how to differentiate between surface hydride and hydroxyl groups with a direct geometry INS spectrometer. On a similar theme, Yamazoe et al. studied hydrogen species on Pd, $\mathrm{Pt}$, and $\mathrm{Rh}$ supported on alumina and carbon black. They showed how the speciation of hydrogen is dependent on the nature of metal and support. Parker et al. investigated the apparent contradictions on the mode of methanol adsorption over HZSM-5e between different techniques, using a combination of INS, infrared spectroscopy and solid-state NMR spectroscopy. It was concluded that at room temperature methanol is very largely associatively adsorbed. When $\mathrm{H}$ species are inside the catalyst, neutron scattering can follow the structural changes during the reaction. Wu and coworkers utilized INS and neutron diffraction combined with first principle simulations to study the structural evolution of a $\mathrm{Ni} / \mathrm{BaH}_{2}$ catalyst during the chemical looping process for ammonia synthesis.

QENS is another neutron technique often used to reveal the motions of surface species during catalysis. Silverwood et al. made use of QENS to study the reaction progress of both a homogeneous reaction - the complexation of $\mathrm{NiCl}_{2}$ with 2,2'-Bipyridine, and a heterogeneous one-water adsorption in chabazite. These reactions illustrated the possibilities of using neutron probes to observe realistic catalytic reactions as they progress. Matam et al. studied methanol dynamics in zeolite H-ZSM-5 at 298, 323, 348, and $373 \mathrm{~K}$ using QENS. They showed that the majority of methanol is immobile, in the range between 70 and $80 \%$, depending on the measurement temperature. Along the same line, O'Malley et al. investigated the dynamics of catechol in zeolite Beta using QENS and molecular dynamics (MD) simulations at $393 \mathrm{~K}$ to understand the behavior of phenolic monomers relevant in the catalytic conversion of lignin via metal nanoparticles supported on zeolites. Two different motions of catechol were suggested in the zeolite based on the MD. Total neutron scattering (TNS) can be used to access the orientation of molecules. Hughes and coworkers studied mixtures of cyclohexane and benzene in both the bulk liquid phase and when confined in MCM-41 mesopores. The bulk mixtures have been studied using TNS, and an integrated TNS and NMR system have studied the confined mixtures. Different molecular orientations in the bulk vs. in the confinement were suggested from the TNS and NMR studies.

As the guest editors, we would like to thank all the authors who contributed to this thematic issue. Their contributions represent excellent examples of the current research trends in the field of neutron scattering for catalysis. We also wish to thank the editor-in-chief, professor Hans-Joachim Freund, for his initiation and encouragement on this special issue and the editorial staff of Topics in Catalysis for their help to organize this issue. We hope that the topics presented in this issue would inspire the readers to further explore the unique power of neutron scattering for catalysis research.

Publisher's Note Springer Nature remains neutral with regard to jurisdictional claims in published maps and institutional affiliations. 